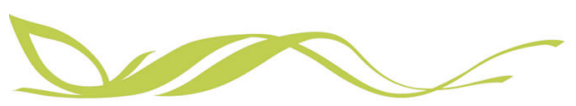

ARTICLE

\title{
Arrival and magnetization of carbonaceous chondrites in the asteroid belt before 4562 million years ago
}

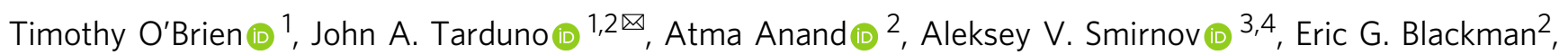
Jonathan Carroll-Nellenback ${ }^{2} \&$ Alexander N. Krot ${ }^{5}$

Meteorite magnetizations can provide rare insight into early Solar System evolution. Such data take on new importance with recognition of the isotopic dichotomy between noncarbonaceous and carbonaceous meteorites, representing distinct inner and outer disk reservoirs, and the likelihood that parent body asteroids were once separated by Jupiter and subsequently mixed. The arrival time of these parent bodies into the main asteroid belt, however, has heretofore been unknown. Herein, we show that weak CV (Vigarano type) and CM (Mighei type) carbonaceous chondrite remanent magnetizations indicate acquisition by the solar wind 4.2 to 4.8 million years after Ca-Al-rich inclusion (CAI) formation at heliocentric distances of $\sim 2-4 \mathrm{AU}$. These data thus indicate that the $\mathrm{CV}$ and $\mathrm{CM}$ parent asteroids had arrived near, or within, the orbital range of the present-day asteroid belt from the outer disk isotopic reservoir within the first 5 million years of Solar System history.

\footnotetext{
${ }^{1}$ Department of Earth and Environmental Sciences, University of Rochester, Rochester, NY 14627, USA. ${ }^{2}$ Department of Physics and Astronomy, University of Rochester, Rochester, NY 14627, USA. ${ }^{3}$ Department of Geological and Mining Engineering and Sciences, Michigan Technological University, Houghton, MI 49931, USA. ${ }^{4}$ Physics Department, Michigan Technological University, Houghton, MI 49931, USA. ${ }^{5}$ Hawai'i Institute of Geophysics and Planetology,

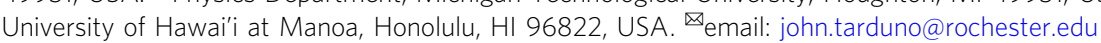


$\mathrm{T}$ he early migration of Jupiter is thought to have resulted in scattering of planetesimals and ultimately the diversity of meteorite types found in the asteroid belt today ${ }^{1-3}$. Magnetic minerals can be created and preexisting grains transformed during aqueous alteration, and such events occurred on the $\mathrm{CM}$ and $\mathrm{CV}$ parent bodies at $\sim 4.8$ and $\sim 4.2$ m.y. after CAI formation, respectively $y^{4,5}$. As we will show using theory and ideal magnetohydrodynamic simulations, magnetizations can be imparted on these minerals by the solar wind, and the recorded field strength can constrain for the first time the heliocentric distance of the parent asteroids at the time of aqueous alteration. However, meteorite magnetizations can also be of intrinsic origin, reflecting the physical nature of magnetic minerals rather than ambient fields ${ }^{6}$, or they can record past parent body core dynamos ${ }^{7-9}$. Prior magnetic work on the CV meteorite Allende has been used to argue for a core dynamo ${ }^{10}$ seemingly overturning more than 50 years of research that held this meteorite as a type example from an undifferentiated asteroid ${ }^{11}$. This paradox must be addressed first before using carbonaceous chondrite meteorite magnetizations to constrain early Solar System events.

Allende was long considered to be a primitive aggregate of CAIs, chondrules, and fine-grained matrix. Subsequently, it was recognized that $\mathrm{CV}$ chondrites had experienced fluid-assisted thermal metamorphism and metasomatic alteration resulting in the formation of secondary minerals ${ }^{12}$, including $\mathrm{Fe}$-sulfides and magnetite important for magnetism. Allende (CV3.6) is one of the most metamorphosed $\mathrm{CV}$ chondrites, having experienced peak temperatures of $\sim 500-600^{\circ} \mathrm{C}^{13}$. Early work recognized that Allende has a magnetization ${ }^{7}$, but only recently has it been claimed that this requires a core dynamo ${ }^{10}$; an interpretation used by some authors as prima facie evidence for differentiation ${ }^{14}$. It is possible to construct thermal models such that $\mathrm{CV}$ chondrites originate in the outermost layers of a partially differentiated asteroid, isolated from interior melting ${ }^{15}$. But no meteorites are known that clearly represent mantle or core samples of the hypothetical differentiated CV asteroid. Achondrite and CV clasts mixed in a breccia are also unknown. Moreover, the estimated initial abundance of ${ }^{26} \mathrm{Al}$ in the $\mathrm{CV}$ parent asteroid, considered to be its major heating source, is insufficient to produce melting and igneous differentiation ${ }^{16}$. Another model suggests that $\mathrm{CV}$ chondrites could represent a "late" accretion ( $~ 1.5$ to 5 m.y. after CAI formation) to a body that had already differentiated ${ }^{17}$, but the lack of differentiated meteorites unambiguously linked to the $\mathrm{CV}$ parent asteroid remains an outstanding question.

\section{Results and discussion}

Paleointensity estimates for Allende reported using varied methods over different ranges of unblocking temperature and coercivity (Supplementary Table S1) span 3 orders of magnitude ( $<1$ to 1600 $\mu \mathrm{T})$. But to be reliable absolute paleointensity recorders, defined here as obeying Thellier's laws ${ }^{8}$ where thermoremanent magnetization theory is applicable, magnetic grains must exhibit single domain or single domain-like ${ }^{18}$ behavior, and they must be noninteracting. Magnetic hysteresis first order reversal curve (FORC) data collected on Allende samples dominated by matrix (see Methods) are strikingly different from those meeting these requirements (Fig. 1a, b and Supplementary Figs. 1-3). Allende FORC data are not dominated by single domain grains but instead show pseudo-single to multidomain behavior with an unusually high coercivity (see Supplementary Information Section 1). While the presence of multidomain grains is a concern, a larger issue regarding recording fidelity is posed by the magnetic interactions seen in the data, which are much stronger than ideal magnetic recorders. However, given the multi-phase magnetic mineralogy of Allende, it is not possible from the magnetic hysteresis data alone to unambiguously identify the source of the interactions and thus its effect on remanence.

Prior studies have concluded that Allende's remanence is carried by an Fe-sulfide; some assert a monoclinic pyrrhotite phase ${ }^{10}$. However, Allende's low field magnetic susceptibility (Methods, Fig. 1c, Supplementary Information Section 1) is dominated by $\mathrm{Fe}-\mathrm{Ni}$ phases (predominantly awaruite, $\mathrm{Ni}_{3} \mathrm{Fe}$ ) and magnetite $\left(\mathrm{Fe}_{3} \mathrm{O}_{4}\right)$, the latter showing evidence for the Verwey transition. This large difference between grains carrying remanence and magnetic susceptibility is unusual in terrestrial rocks known to be reliable paleointensity recorders. When cycled from low to successively higher temperatures, through the temperature range carrying the remanence, magnetic susceptibility data define the growth of an anomaly between $\sim 200$ and $270^{\circ} \mathrm{C}$ (Fig. 1d), during which Allende increases its capacity to record an applied magnetic field. On cooling, a portion of this increased capacity is retained. These changes are reminiscent of the lambda transition in hexagonal pyrrhotite: weakly magnetic antiferromagnetic pyrrhotite changes to a strongly magnetic ferrimagnetic state through vacancy reordering ${ }^{8}$.

To explore directly the apparent unblocking temperature interval carrying magnetic remanence, a partial thermoremanent magnetization (pTRM) was imparted, thermally demagnetized, and re-imparted in cycles (Fig. 1e; Supplementary Information Section 2). Contrary to predictions, subsequent pTRMs result in higher intensities. Repeatable near zero values after demagnetization argue against laboratory thermal alteration (although this is sub-sample dependent; Supplementary Fig. 4). Instead, this behavior appears to be the remanence equivalent of magnetic susceptibility cycling (Fig. 1d) results where the material increases systematically its magnetization capability. We also observe nonsystematic remanence increases or decreases (Supplementary Fig. 4) in zero field heating of pTRMs applied at higher temperatures $\left(620\right.$ to $\left.490{ }^{\circ} \mathrm{C}\right)$, also contrary to predictions but consistent with strong magnetic interactions.

Allende remanence data shows evidence for multiple components of magnetization (Supplementary Fig. 5), which fall mainly in one quadrant. A fusion crust test that would exclude terrestrial contamination of Allende data ${ }^{10}$ is ambiguous because low unblocking temperature magnetizations (natural remanent magnetization to $190{ }^{\circ} \mathrm{C}$ ) within millimeters of the crust are indistinguishable from the key interior magnetizations (Supplementary Fig. 5, Supplementary Information Section 3). While composed of multiple components, it has nevertheless been proposed that the remanence is "quasi-single" component in nature and that it was acquired over millions of years, incompatible with a time-varying external source ${ }^{10}$. This interpretation assumes that magnetic unblocking during laboratory demagnetization is equivalent to magnetic acquisition (blocking) in nature. To test this equivalence, we heated Allende samples and changed the polarity of an applied field during cooling (see Methods, Supplementary Fig. 6, Supplementary Information Section 4). This treatment reproduces the "quasi-single" component magnetization (Fig. 1f). In contrast, experiments on terrestrial basalt samples differ, revealing imparted reversals (Supplementary Fig. 7, Supplementary Information Section 4). These experiments indicate that Allende magnetic carriers lock in the field to which they are first exposed during cooling and positive interactions during subsequent cooling result in a "quasi-single" component remanence. The lack of evidence for acquisition over long time intervals suggests that impacts ${ }^{19,20}$ remain a potential source for Allende's magnetization.

Energy dispersive X-ray spectroscopy and electron microprobe data (see Methods, Supplementary Information Section 5), show that FeNi-metal grains commonly occur in close association with FeNi-sulfides and Fe-sulfides (Fig. 1g, h, Supplementary Fig. 8), 
a

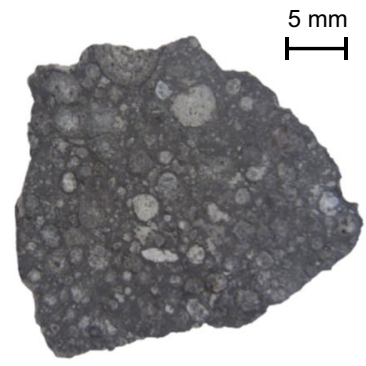

b

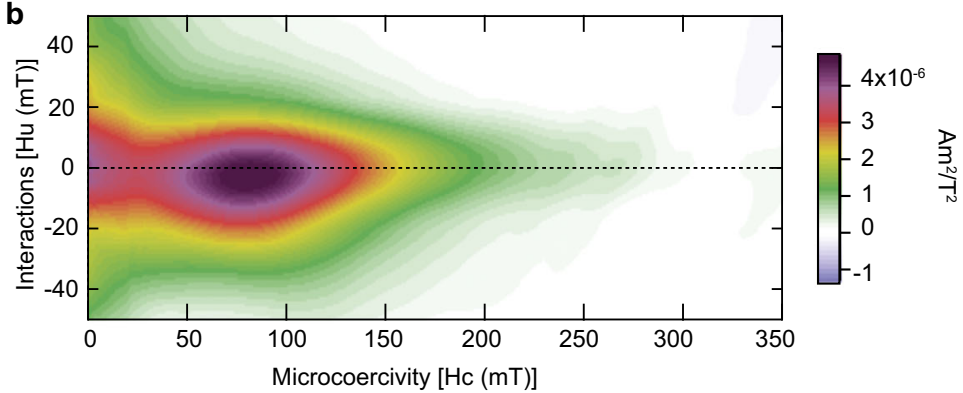

c
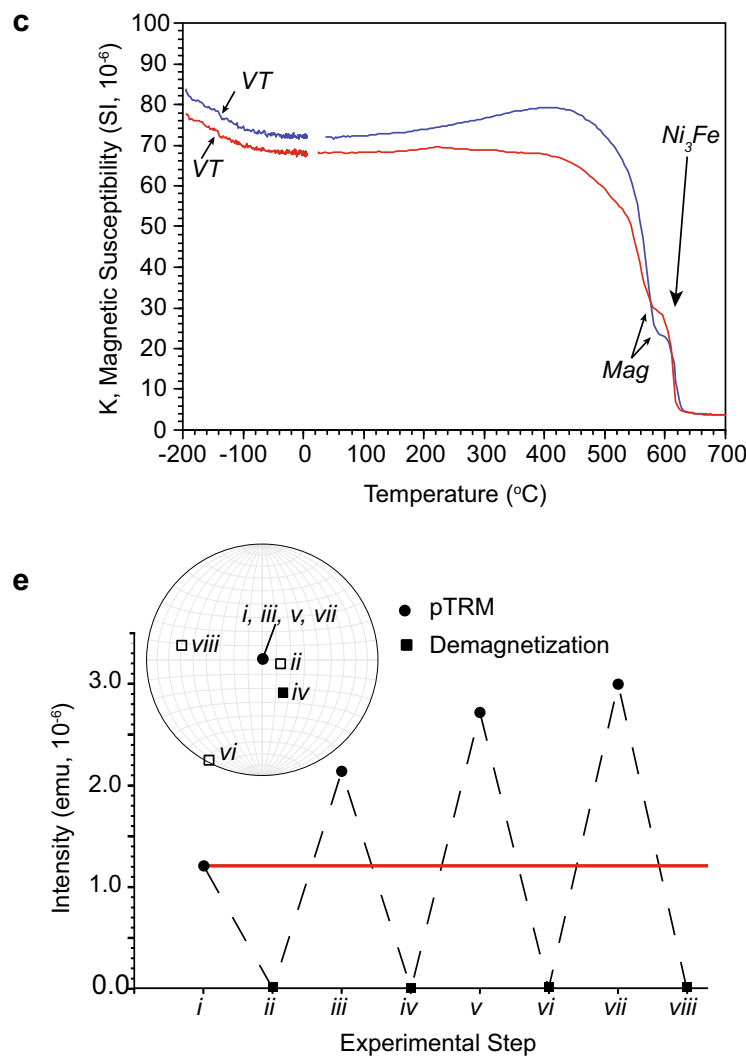

g

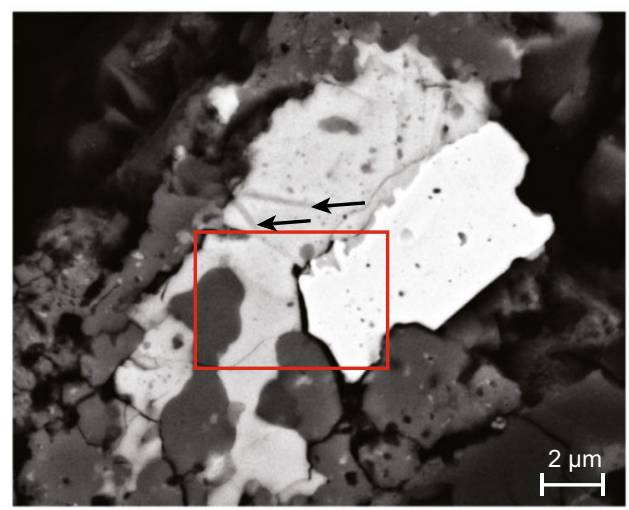

d

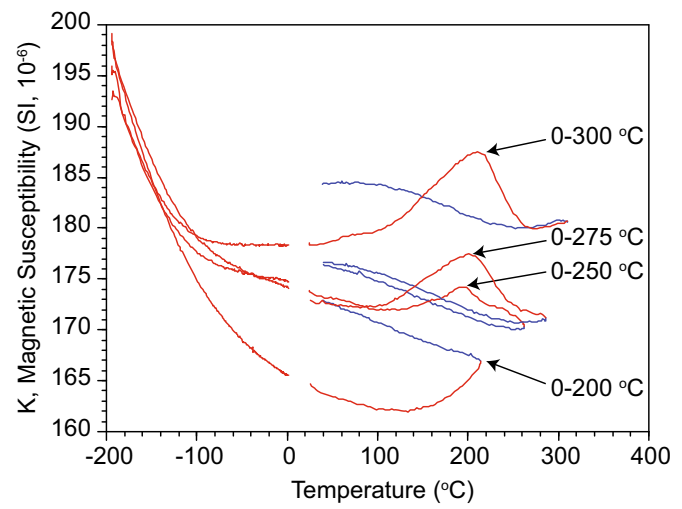

f

AM2UBB02

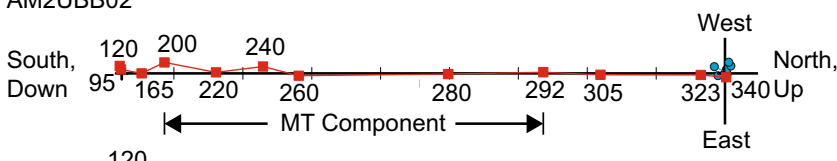
120 h
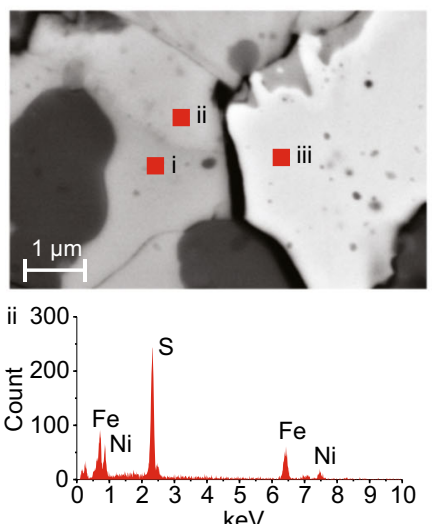
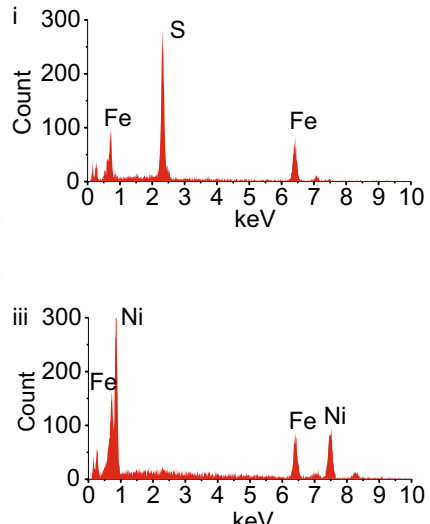

providing mineralogical context for magnetic interactions. We interpret fine textures as reflecting complex exsolution between low $\mathrm{Ni}$ pentlandite ${ }^{21}$ and pyrrhotite (Fig. 1g, h). Exchange interaction between monoclinic and hexagonal pyrrhotite phases formed during exsolution may explain Allende's rock magnetic behavior, including the apparent high coercivity seen in the FORC analyses (Supplementary Fig. 8, Supplementary
Information Section 6). Regardless of the exact interaction mechanism, the very evidence for profound interactions, as well as the evidence for reordering indicates that Allende lacks paleointensity recording requirements and that the prior nominal value $^{10}$ of $\sim 20 \mu \mathrm{T}$, as well as other numbers (Table S1) have no basis in paleointensity estimation otherwise solidly founded on the physics of the magnetization process ${ }^{8}$. Importantly, this also 
Fig. 1 Rock magnetism, electron microscopy and tests of Allende paleomagnetic recording fidelity. a Typical Allende sample analyzed. $\mathbf{b}$ First-order reversal curve (FORC, a measure of magnetic interaction Hu versus microcoercivity $\mathrm{Hc}$ ) showing large interactions (correspondingly large spread of Hu values; compare with ideal single domain case (Supplementary Fig. 1b) where Hu is restricted and the central FORC peak is more than order of magnitude greater than that seen in Allende data). For FORC parameters, see Supplementary Fig. 2. c Magnetic susceptibility versus temperature in an Ar atmosphere. VT, Verwey transition; mag, magnetite. $\mathbf{d}$ Magnetic susceptibility cycling at successively higher temperatures measured in an Ar atmosphere. Red: heating, Blue: cooling. e Partial thermal remanent magnetization (pTRM) between $292^{\circ} \mathrm{C}$ and room temperature (i, iii, v, vii) followed by demagnetization (ii, iv, vi, viii). All experiments conducted in an Ar atmosphere. Red horizontal line: predicted values. $\mathbf{f}$ Orthogonal vector plot of demagnetization of remanence acquired after cooling from $340{ }^{\circ} \mathrm{C}$ in a reversing applied magnetic field (polarity changes illustrated by bar chart). Field applied along $\mathrm{z}$ axis; Red: inclination. Blue: declination. Experiments in Ar; temperature labels are ${ }^{\circ} \mathrm{C}$. Interval of medium temperature component (MT) interpreted in prior work as holding a primary remanence highlighted. $\mathbf{g}$ Backscatter scanning electron microscope image; red box show enlargement area (h). Arrows highlight exsolution textures. h Energy dispersive spectroscopy analyses: i. Fe-sulfide, ii. Fe-Ni sulfide and iii. Ni-Fe metal.

extends to non-thermal mechanisms for estimating paleointensity which arises from a TRM in Allende (see Supplementary Information Section 6). Instead, previous values are physical property measurements of intrinsic magnetic interactions. Thus, we conclude that Allende's magnetism is compatible with an undifferentiated parent body ${ }^{11,16}$, but that it cannot otherwise be used to shed light on ambient fields in the early Solar System.

Paleointensity analyses of the CV chondrite Kaba (CV3.1), however, are viable given the single domain-like (i.e., pseudosingle domain) magnetic carriers of magnetite composition ${ }^{22}$, without the extreme interaction and reordering problems when the dominant remanence carrier is a multiphase pyrrhotite (Supplementary Information Section 7). Kaba samples are dominated by viscous remanent magnetizations (VRMs) ${ }^{22}$, but the analysis that appears least affected by VRM yields a field of $\sim 0.4-1.1 \mu \mathrm{T}$. This is similar to that obtained from CM carbonaceous chondrites which have remanences carried by magnetite (i.e., Murchison, paleointensity estimate of approximately $\sim 1 \mu \mathrm{T}$ derived in two independent studies ${ }^{23,24}$, see Supplementary Information Section 7). We explore below whether external fields can explain these values.

CV asteroid metasomatism occurred at $\sim 4562$ million years ago $(\mathrm{Ma})^{5}, \sim 4.2_{-0.7}^{+0.8}$ m.y. after CV CAIs and textural evidence indicates magnetite formation was relatively rapid (Supplementary Information Section 7 ). This is $\sim 1.5$ m.y. after cooling of volcanic angrites from the inner disk isotopic reservoir in a field $<0.6 \mu \mathrm{T}^{25}$ interpreted to record magnetization after nebular dispersal which otherwise might be a magnetization source ${ }^{26}$. Solar wind ram pressure was much higher at these times due to the initial faster spin of the Sun, which in turn is associated with greater magnetic activity and higher rates of mass loss ${ }^{27}$. If the gyro-radius of the stellar wind ions is small compared to the asteroid, field can pile up given obstacles to slow the solar wind, expected for early solar system conditions (Supplementary Information Section 8). The ion gyro-radius scales as $r_{g} \sim$ $250 \mathrm{~km}\left(B_{0,1 \mathrm{AU}} / 4 n T\right)^{-1}$ where $B_{0,1 \mathrm{AU}}$ is the field at $1 \mathrm{AU}$. This condition is not met for the present day solar wind. For solar-type stars 100 times younger, however, the product of surface field and rotation rate can be $>20$ times larger ${ }^{28,29}$, increasing the toroidal field at $1 \mathrm{AU}$ by this factor in a Parker spiral model, and reducing $r_{g}$ to $<12.5 \mathrm{~km}$. Correspondingly, the ratio of the Bohm diffusion time to wind crossing time for an asteroid of radius $r_{b} \sim 20 r_{g}$ satisfies $\quad t_{\text {dif }} / t_{w} \geq\left(20 r_{g}\right)^{2} /\left(c_{s} r_{g}\right)\left(v_{w} / 20 r_{g}\right) \sim 20\left(v_{w} / c_{s}\right) \sim 100$ assuming a wind sound speed $c_{s}=100 \mathrm{~km} \mathrm{~s}^{-1}$, and wind velocity $v_{w}=500 \mathrm{~km} \mathrm{~s}^{-1}$. Under these circumstances, the flow may advect and drape the field around the asteroid.

As long as the magnetic diffusivity of the body $v_{b}$ is small enough, the field can accumulate near the surface until the magnetic force density (the sum of magnetic pressure and tension) balances that from the incoming wind ram pressure ${ }^{30}$. The amplification factor, expressed as the ratio of the maximum amplified field strength in the body $B_{0}$ to the incoming wind field strength $B_{i}$ that we expect and confirm by our simulations is $A \equiv B_{0} / B_{i} \simeq\left(1+\chi_{v}\right) \max \left\{1, \min \left[M \sqrt{\gamma \beta}, R_{m, b}\right]\right\} \simeq M \sqrt{\gamma \beta}=$ $(8 \pi \rho)^{1 / 2} v_{w} / B_{i}$. Here $\rho$ is the wind mass density, $\gamma$ is the constant pressure to constant volume heat capacity ratio $(\gamma=5 / 3$, for adiabatic and $\gamma=1$ isothermal) and the last two relations follow when the volume magnetic susceptibility $\chi_{v} \sim 0$ and the magnetic Reynolds number associated with the body satisfies $R_{m, b} \equiv v_{w} r_{b} / \nu_{b}>M \sqrt{\gamma \beta}>1$, where $M \equiv v_{w} / c_{s}$ is the wind Mach number and $\beta$ is the ratio of wind thermal pressure to wind magnetic pressure. The above expression for $A$ can be immediately simplified into an expression for the amplified field

$$
\begin{aligned}
B_{0} & =v_{w}\left(1+\chi_{v}\right)(8 \pi \rho)^{1 / 2} \\
& =2.1 \mu \mathrm{T}\left(1+\chi_{v}\right)\left(v_{w} / 1000 \mathrm{~km} \mathrm{~s}^{-1}\right)\left(n / 1000 \mathrm{~cm}^{-3}\right)^{1 / 2},
\end{aligned}
$$

where we have scaled the wind speed and number density $n$ (assuming a hydrogen dominated wind) to values possible for an early solar wind (1-5 Myr-old) for which the average $\mathrm{x}$-ray luminosity, mass loss rates, and ram pressure could be $10^{4}$ times larger ${ }^{30,31}$ than for the present Sun. A giant stellar flare could also transiently increase the wind ram pressure by another factor of $\sim 10$, increasing $B_{0}$ by another factor of 3 .

Using ideal 3D magnetohydrodynamic (MHD) simulations (Fig. 2, Methods, Supplementary Information Section 8, and Movie S1) for $\chi_{v}=0$ we observe high amplification factors $A \approx 12.25 \sim 1.07 M \sqrt{\gamma \beta}$, confirming the scaling relations above. The latter similarity implies that equation (1), which depends only on the assumed wind speed and wind particle density and was derived from an amplification factor of exactly $M \sqrt{\gamma \beta}$, is a good estimate for the field amplified at the surface of the asteroid predicted by the simulations.

The CV magnetization constraints (both field strength and directions, see Supplementary Information Section 8) can be met at orbital distances consistent with the current asteroid belt $(\sim 2-4$ AU) by these amplified solar wind induced fields, or by smaller amplifications and orbital distances (we view the former as more likely) (Fig. 3), representing the first direct tracking of the heliocentric distance of a meteorite parent body using paleomagnetism ${ }^{32,33}$. Aqueous alteration on the CM parent body occurred at $\sim 4.8$ m.y. after CAI formation ${ }^{4}$, essentially contemporaneous with $\mathrm{CV}$ metasomatism. CM magnetizations are also satisfied at $\sim 2-4 \mathrm{AU}$, but not at much greater distances.

The arrival of the $\mathrm{CV}$ and $\mathrm{CM}$ parent bodies at or within heliocentric distances of the asteroid belt from an outer disk isotopic reservoir by the magnetization age of ca. 4562 Ma places further constraints on early Solar System events. Prior to disk dispersal, the accretion stream onto the Sun would have shielded asteroids from the solar wind, possibly resulting in even smaller field values. Accretion rates drop rapidly after giant planets have opened $>\sim 1$ AU-scale gaps (transitional disks), and the Solar System must have gone through this phase shortly after the formation of Jupiter and Saturn. Once accretion rates fall 


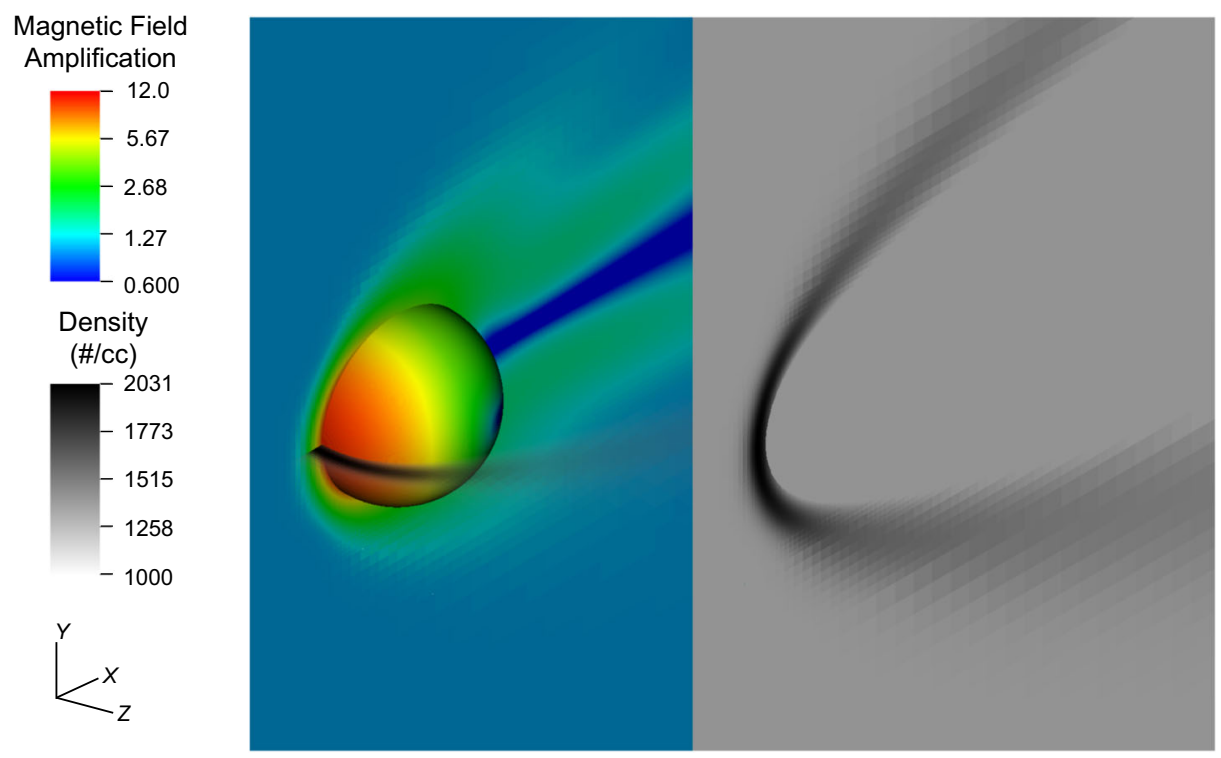

Fig. 2 Magnetohydrodynamic numerical simulation results. Magnetic field amplification (color in logscale) along xy midplane and immediately above surface of asteroid (see Methods). Grayscale shows the number density (linear scale) in xz midplane as well as along xy midplane (transposed for visibility).

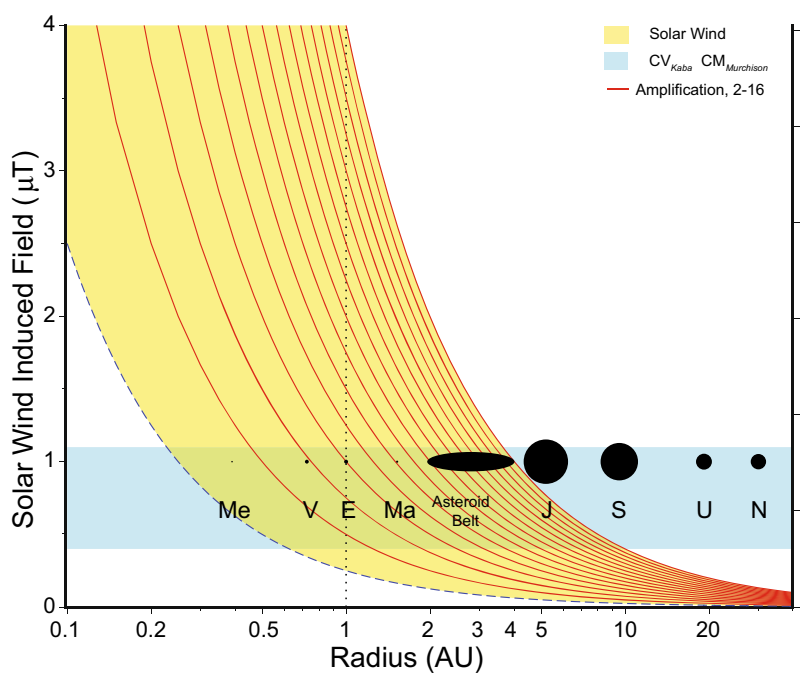

Fig. 3 Solar wind interactions with young solar system asteroids. Solar wind induced field versus radius of orbit. Dashed line: minimum field limit $(0.25 \mu \mathrm{T}$ at $1 \mathrm{AU})$. Red lines: field amplified by factors (A) between 2 and 16. Yellow region: range of fields capable of magnetizing $C V$ and $C M$ chondrites as magnetic minerals either cool through their Curie temperature or grow through a blocking volume during metasomatism. Blue: $\mathrm{CV}_{\text {Kaba }}$ and $\mathrm{CM}_{\text {Murchison }}$ are select paleointensity values from the Kaba and Murchison meteorites (see text and Supplementary Information Section 7). Present-day location of planets and asteroid belt shown diagrammatically for reference: Me, Mercury; V, Venus; E, Earth; Ma, Mars; J, Jupiter; S, Saturn; U, Uranus; N, Neptune.

below $\sim 10^{-10}$ Solar mass $\mathrm{yr}^{-1}$, dissipation of gas and dust by photoevaporation, aided by stellar winds, is very rapid $^{34}$. CV and $\mathrm{CM}$ external solar wind magnetizations thus indicate that accretion rates had fallen below this threshold, and that gas and dust were essentially dissipated, before 4562 Ma.

\section{Methods}

Experiments were conducted at the University of Rochester Paleomagnetic Laboratories (UR) and the Michigan Technological University Earth Magnetism
Laboratory (MTU). Three separate Allende samples were studied; two were obtained commercially and one from a university collection. Subsamples analyzed were at least $1 \mathrm{~cm}$ from the fusion crust. Magnetic susceptibility data were collected at both the UR and MTU labs using an AGICO KLY-4S Spinner Kappabridge equipped with a CS-3 Furnace Apparatus and a CS-L Cryostat and an AGICO MFK1-FA Kappabridge equipped with a CS-3 Furnace Apparatus and a CS-L Cryostat, respectively. Magnetic hysteresis data, including FORC analyses ${ }^{35}$ and were collected using a Princeton Measurement Corporation Model 2900 Alternating Gradient Force Magnetometers. For all magnetic hysteresis and remanence measurements 1-2 mm-sized un-oriented subsamples were selected to target matrix material and exclude large visible chondrules, (minimizing the effects of inverse TRM recently reported from a study of Allende chondrules ${ }^{36}$ ). A 3 component $2 \mathrm{G}$ DC SQUID magnetometer with high resolution sensing pickup coils access bore 4.2 $\mathrm{cm}$, sensitivity $\sim 5 \times 10^{-12} \mathrm{~A} \mathrm{~m}^{2}$ ) in the UR magnetically shielded room (ambient field $<200 \mathrm{nT}$ ) was used for remanence measurements. Thermal demagnetization and PTRM acquisition experiments were conducted using a $\mathrm{CO}_{2}$ laser $^{37-40}$ in conjunction with a new controlled atmosphere chamber (e.g. Argon or Nitrogen) and additional magnetic shielding to produce a magnetically null environment (for field-off demagnetization) or an applied field coil (for field-on experiments). The non-magnetic atmosphere chamber is outfitted with a $\mathrm{ZnSe}$ window that permits transmission of the $\mathrm{CO}_{2}$ laser beam, while maintaining the gas atmosphere within the chamber during thermal experiments. Allende samples were mounted on fused quartz rods with sodium silicate and minor amounts of Omega cement.

Low to intermediate temperature $\left(0-292^{\circ} \mathrm{C}\right)$ pTRM experiments involved cycles, in either air, argon or nitrogen of the application of a pTRM at $292^{\circ} \mathrm{C}$ in a $30 \mu \mathrm{T}$ field, followed by a heating to $292{ }^{\circ} \mathrm{C}$ in the absence of a field. In high to intermediate temperatures $\left(620-490{ }^{\circ} \mathrm{C}\right)$ pTRM experiments, a pTRM was imparted by heating to $620^{\circ} \mathrm{C}$ in the absence of a field and cooling in a $30 \mu \mathrm{T}$ field to $490^{\circ} \mathrm{C}$, after which the applied field was turned off and the sample was cooled to room temperature. The sample was subsequently heated to $292{ }^{\circ} \mathrm{C}, 310^{\circ} \mathrm{C}$, and 490 ${ }^{\circ} \mathrm{C}$ in zero field. An infrared pyrometer (FLIR ThermaCam SC640) was used to measure cooling curves for Allende samples and a terrestrial basalt. The basalt sample is a clast in a hyaloclastite (Ocean Drilling Program Leg 197, Hole 1206A7R-1, Int. 128-130 cm $)^{41,42}$. Calibration samples were heated with a $\mathrm{CO}_{2}$ laser outside of the controlled atmosphere chamber and cooled with Ar flowing across the face of the sample to simulate flow of argon during the experiments. Allende and basalt samples were heated to initial temperatures of $340{ }^{\circ} \mathrm{C}$ and $260{ }^{\circ} \mathrm{C}$, respectively, to ensure that enough time was spanned during cooling to apply at least one field reversal within the remanence carrier unblocking range. A switch was constructed to allow an instant transition between field polarities. The applied field strength for all experiments was $60 \mu \mathrm{T}$ and all heatings were in Ar. To limit potential alteration, Allende samples were not demagnetized prior to imparting the pTRM; the peak pTRM temperature of $340{ }^{\circ} \mathrm{C}$ however ensures that dominant remanence has been demagnetized. Basalt samples were demagnetized prior to the pTRM step to evaluate unblocking temperature range of remanence carriers for the samples.

Scanning electron microscopy (SEM) and energy dispersive spectroscopy (EDS) analyses were conducted using a Zeiss Auriga Scanning Electron Microscope with an EDAX spectrometer at the University of Rochester Integrated Nanosystems 
Center. Samples were prepared in an acrylic mount and given a final polished surface using colloidal silica prior to mounting and carbon coating for SEM analyses. Additional analyses targeting the Fe-Ni-S system were made using a Cameca SX-100 electron microprobe at Rensselaer Polytechnic Institute's Electron Microprobe Laboratory. The microprobe was calibrated to measure S, Fe, O, Ni, and $\mathrm{Si}$ using the following respective standards: $\mathrm{FeS}_{2}$ (2 standards), magnetite $\left(\mathrm{Fe}_{3} \mathrm{O}_{4}\right)$, nickel, and kyanite. Data were collected with an accelerating voltage of 15 $\mathrm{keV}$ and $10 \mathrm{keV}$.

We investigated a magnetized solar wind overrunning an asteroid with the Astro$\mathrm{BEAR}^{43}$ adaptive mesh-refinement code. The young stellar wind was given a density of 1000 particles $\mathrm{cc}^{-1}$, a temperature of $10^{6} \mathrm{~K}$, and was traveling at $500 \mathrm{~km} \mathrm{~s}^{-1}$ (in $x$ ) carrying a perpendicular magnetic field of $100 \mathrm{nT}$ (in the $y$ direction) into a magnetized ambient at the same density and temperature. The stellar wind had a Mach number of 4.74, a beta of 3.47, and an Alfvenic Mach number of 8 . We used the equations of resistive MHD with a specific heat ratio of 1.667 to model the wind as a monoatomic gas. The asteroid was modeled as a $500 \mathrm{~km}$ radius solid boundary centered at the origin, which does not act as a significant source of particles for flows diverging from the surface. The asteroid has a conductive outer layer, i.e., its $10 \%$ outer shell on the day side is 10 times more conductive than the parent body, to simulate a cometary-like solar wind interaction (see Supplementary Information Section 8). Diffusion was turned on for all flows outside the asteroid to speed up convergence. The solution results can be rescaled to arbitrary asteroid radius provided the asteroid radius > gyration radius. The wind density, temperature, velocity, and magnetization can be rescaled provided the same magnetic beta and Mach number are used. The simulation boundaries are extrapolated and sufficiently far away, such that the magnetic field is perpendicular as it leaves the box. The base resolution was $64 \times 64 \times 64$ with 4 additional levels of adaptive mesh refinement (AMR) around the asteroid allowing us to resolve the asteroid's diameter with 128 zones.

\section{Data availability}

Data are available on the Earthref.org MagIC database (earthref.org/MagIC/16980).

Received: 4 June 2020; Accepted: 27 October 2020;

Published online: 04 December 2020

\section{References}

1. Warren, P. H. Stable-isotopic anomalies and the accretionary assemblage of the Earth and Mars: a subordinate role for carbonaceous chondrites. Earth Planet Sci. Lett. 311, 93-100 (2011).

2. Kruijer, T. S., Kleine, T. \& Borg, L. E. The great dichotomy of the early Solar System. Nat. Astron. 4, 32-40 (2020).

3. DeMeo, F. E. \& Carry, B. Solar system evolution from compositional mapping of the asteroid belt. Nature 505, 629-634 (2014).

4. Fujiya, W., Sugiura, N., Hotta, H., Ichimura, K. \& Sano, Y. Evidence for the late formation of hydrous asteroids from young meteoritic carbonates. Nat. Commun. 3, 627 (2012).

5. Doyle, P. M. et al. Early aqueous activity on the ordinary and carbonaceous chondrite parent bodies recorded by fayalite. Nat. Commun. 6, 7444 (2015).

6. Brecher, A. \& Albright, L. The thermoremanence hypothesis and the origin of magnetization in iron meteorites. J. Geomag. Geoelec. 29, 379-400 (1977).

7. Banerjee, S. K. \& Hargraves, R. B. Natural remanent magnetizations of carbonaceous chondrites and the magnetic field in the early solar system. Earth Planet Sci. Lett. 17, 110-119 (1972).

8. Dunlop, D. J. \& Özdemir, Ö. Rock magnetism, Fundamentals and Frontiers. (Cambridge Univ. Press, Cambridge, UK, 1997).

9. Tarduno, J. A. et al. Evidence for a dynamo in the main group pallasite parent body. Science 338, 939-942 (2012).

10. Carporzen, L. et al. Magnetic evidence for a partially differentiated carbonaceous chondrite parent body. Proc. Natl Acad. Sci. USA 108, 6386-6389 (2011).

11. Scott, E. R. D. \& Krot, A. N. Chondrites and their components. In Treatise on Geochemistry: Meteorites and Cosmochemical Processes 1 (eds. Holland, H., Turekian, K.) 65-137 (Elsevier, Oxford, UK, 2014).

12. McSween, H. Y. Jr. Petrographic variations among carbonaceous chondrites of the Vigarano type. Geochim. Cosmochim. Acta 41, 1777-1790 (1977).

13. Brearley, A. J. Origin of graphitic carbon and pentlandite in matrix olivines in the Allende meteorite. Science 285, 1380-1382 (1999).

14. Weiss, B. P. \& Elkin-Tanton, L. T. Differentiated planetesimals and parent bodies of chondrites. Annu. Rev. Earth Planet Sci. 41, 529-560 (2013).

15. Elkin-Tanton, L. T., Weiss, B. P. \& Zuber, M. T. Chondrites as samples of differentiated planetesimals. Earth Planet Sci. Lett. 305, 1-10 (2011).

16. Nagashima, K., Krot, A. N. \& Komatsu, M. ${ }^{26}$ Al- ${ }^{26} \mathrm{Mg}$ systematics in chondrules from Kaba and Yamato 980145 CV3 chondrites. Geochim. Cosmochim. Acta 201, 303-319 (2017).
17. Johansen, A., MacLow, M.-M., Lacerda, P. \& Bizzarro, M. Growth of asteroids, planetary embryos, and Kuiper belt objects by chondrule accretion. Sci. Adv. 1, 1500109 (2015).

18. Almeida, T. P. et al. Direct visualization of the thermomagnetic behavior of pseudo-single-domain magnetite particles. Sci. Adv. 2, e1501801 (2016).

19. Bland, P. A. et al. Pressure-temperature evolution of primordial solar system solids during impact-induced compaction. Nat. Commun. 5, 5451 (2014).

20. Muxworthy, A. R. et al. Evidence for an impact-induced magnetic fabric in Allende, and exogenous alternatives to the core dynamo theory for Allende magnetization. Meteoritics Planet Sci. 52, 2132-2146 (2017).

21. Funaki, M. \& Wasilewski, P. A relation of magnetization and sulfidization in the parent body of the Allende (CV3) carbonaceous chondrite. Meteorit. Planet Sci. 34, A39 (1999)

22. Gattacceca, J., Weiss, B. P. \& Gounelle, M. New constraints on the magnetic history of the CV parent body and the solar nebula from the Kaba meteorite. Earth Planet Sci. Lett. 455, 166-175 (2016).

23. Cournede, C. et al. An early solar system magnetic field recorded in CM chondrites. Earth Planet Sci. Lett. 410, 62-74 (2015).

24. Kletetschka, G., Kohout, T. \& Wasilewski, P. J. Magnetic remanence in the Murchison meteorite. Meteorit Planet Sci. 38, 399-405 (2003).

25. Wang, $\mathrm{H}$. et al. Lifetime of the solar nebula constrained by meteorite paleomagnetism. Science 355, 623-627 (2017).

26. Urrutia-Fucugauchi, J., Pérez-Cruz, L. \& Flores-Gutiérrez, D. Meteorite paleomagnetism - From magnetic domains to planetaryfields and core dynamos. Geofísica Intern 53, 343-363 (2014).

27. Tarduno, J. A., Blackman, E. G. \& Mamajek, E. E. Detecting the oldest geodynamo and attendant shielding from the solar wind: Implications for habitability. Phys. Earth Planet Inter 233, 68-87 (2014).

28. Johnstone, C. P., Güdel, M., Brott, I. \& Lüftinger, T. Stellar winds on the main sequence - II. The evolution of rotation and winds. Astron. Astrophys. 577, 1-22 (2015)

29. Blackman, E. G. \& Owen, J. E. Minimalist coupled evolution model for stellar $\mathrm{X}$-ray activity, rotation, mass loss, and magnetic field. Month Not. R. Astron Soc. 458, 1548-1558 (2016).

30. O'Fionnagáin, D. \& Vidotto, A. A. The solar wind in time: a change in the behaviour of older winds? Month Not. R. Astron. Soc. 476, 2465-2475 (2018).

31. Brown, I. G., Watson-Munro, C. N. \& Gold, T. Laboratory simulation of magnetic fields produced in the moon by the trapped field of the solar plasma stream. Phys. Lett. 20, 631-632 (1996).

32. Tarduno, J. A., O'Brien, T. M. \& Smirnov, A. V. Does the magnetization of CV meteorites record a parent body core dynamo? Lunar Planet Sci. Conf. 47, 2609 (2016)

33. Tarduno, J. A., O’Brien, T. M., Blackman, E. G. \& Smirnov, A. V. Magnetization of CV meteorites in the absence of a parent body core dynamo. Lunar Planet Sci. Conf. XLVIII, 2850 (2017).

34. Alexander, R., Pascucci, I., Andrews, S., Armitage, P., Cieza, L. The Dispersal of Protoplanetary Disks. in Protostars and Planets VI (eds. Beuther, H., Klessen, R., Dullemond, C.P., Henning, T.) 475-496 (Univ. of Arizona Press, Tucson, AZ USA, 2014)

35. Roberts, A. P., Pike, C. R. \& Verosub, K. L. FORC diagrams: a new tool for characterizing the magnetic properties of natural samples. J. Geophys. Res. 105, 28,461-28,475 (2000).

36. Kletetschka, G. Magnetization of extraterrestrial Allende material may relate to terrestrial descend. Earth Planet Sci. Lett. 487, 1-8 (2018).

37. Tarduno, J. A., Cottrell, R. D., Watkeys, M. K. \& Bauch, D. Geomagnetic field strength 3.2 billion years ago recorded by single silicate crystals. Nature 446 , 657-660 (2007)

38. Tarduno, J. A., Cottrell, R. D., Davis, W. J., Nimmo, F. \& Bono, R. K. A Hadean to Paleoarchean geodynamo recorded by single zircon crystals. Science 349, 521-524 (2015)

39. Bono, R. K., Tarduno, J. A., Nimmo, F. \& Cottrell, R. D. Young inner core inferred from Ediacaran ultra-low geomagnetic field intensity. Nat. Geosci. 12, 143-147 (2019).

40. Tarduno, J. A. et al. Paleomagnetism indicates that primary magnetite in zircon records a strong Hadean geodynamo. Proc. Natl Acad. Sci. USA 117, 2309-2318 (2020).

41. Tarduno, J. A., Duncan, R. A. \& Scholl, D. W. Shipboard Scientific Party, Proc ODP, Init. Repts. (Ocean Drilling Program, College Station, TX), 197 (2002).

42. Tarduno, J. A. et al. The Emperor Seamounts: Southward motion of the Hawaiian Hotspot plume in Earthas mantle. Science 301, 1064-1069 (2003).

43. Cunningham, A. J., Frank, A., Varniére, P., Mitran, S. \& Jones, T. W. Simulating magnetohydrodynamical flow with constrained transport and adaptive mesh refinement: Algorithms and tests of the AstroBEAR code. Astrophys J. Suppl. Series 182, 519-542 (2009).

\section{Acknowledgements}

We acknowledge the A.E. Seaman Museum for use of Allende specimen AL-1357, and Eric Twelker for Allende samples AM12-7 and AM12-24. This work was supported by 
NASA grant NNX14AI20G and NSF grant EAR1039846 to J.A.T. E.G.B. acknowledges support from grants HST-AR-13916.002 and NSF-AST1515648 and the Kavli Institute for Theoretical Physics (USCB) supported by NSF grant PHY-1125915. A.N.K. acknowledges support from NASA grant NNX17AE22G.

\section{Author contributions}

J.A.T. conceived of and led the investigation. Paleomagnetic experiments and electron microscope analyses were carried out by T.O. and data analyzed by T.O. and J.A.T. Rock magnetic analyses were performed by T.O. and A.S. and interpreted by T.O., A.S. and J.A.T. A.S. contributed the rock magnetic interaction model. E.G.B. provided constraints on solar wind interactions based on theory. J.C.-N. and A.A. conducted MHD simulations, with input from E.B. and J.A.T. A.N.K. contributed meteoritic interpretations. J.A T. and T.O. wrote the first draft of the main text and supplement, respectively, and all authors contributed to the final manuscript.

\section{Competing interests}

The authors declare no competing interests.

\section{Additional information}

Supplementary information is available for this paper at https://doi.org/10.1038/s43247 020-00055-w.

Correspondence and requests for materials should be addressed to J.A.T.
Peer review information Primary handling editor: Joseph Aslin.

Reprints and permission information is available at http://www.nature.com/reprints

Publisher's note Springer Nature remains neutral with regard to jurisdictional claims in published maps and institutional affiliations.

(c) (i)

Open Access This article is licensed under a Creative Commons Attribution 4.0 International License, which permits use, sharing, adaptation, distribution and reproduction in any medium or format, as long as you give appropriate credit to the original author(s) and the source, provide a link to the Creative Commons license, and indicate if changes were made. The images or other third party material in this article are included in the article's Creative Commons license, unless indicated otherwise in a credit line to the material. If material is not included in the article's Creative Commons license and your intended use is not permitted by statutory regulation or exceeds the permitted use, you will need to obtain permission directly from the copyright holder. To view a copy of this license, visit http://creativecommons.org/ licenses/by/4.0/.

(c) The Author(s) 2020 